\title{
Design of Tunnel Magnetoresistive-Based Circular MFL Sensor Array for the Detection of Flaws in Steel Wire Rope
}

\author{
Liu Xiucheng, Wang Yujue, Wu Bin, Gao Zhen, and He Cunfu \\ College of Mechanical Engineering and Applied Electronics Technology, Beijing University of Technology, Beijing, China \\ Correspondence should be addressed to Liu Xiucheng; xiuchliu@bjut.edu.cn
}

Received 27 January 2016; Revised 24 July 2016; Accepted 15 August 2016

Academic Editor: Eugenio Martinelli

Copyright ( 2016 Liu Xiucheng et al. This is an open access article distributed under the Creative Commons Attribution License, which permits unrestricted use, distribution, and reproduction in any medium, provided the original work is properly cited.

\begin{abstract}
Tunnel magnetoresistive (TMR) devices have superior performances in weak magnetic field detection. In this study, TMR devices were first employed to form a circular magnetic flux leakage (MFL) sensor for slight wire rope flaw detection. Two versions of this tailor-made circular TMR-based sensor array were presented for the inspection of wire ropes with the diameters of $14 \mathrm{~mm}$ and $40 \mathrm{~mm}$, respectively. Helmholtz-like coils or a ferrite magnet-based magnetizer was selected to provide the proper magnetic field, in order to meet the technical requirements of the TMR devices. The coefficient of variance in the flaw detection performance of the sensor array elements was experimentally estimated at $4.05 \%$. Both versions of the MFL sensor array were able to detect multiple single-broken wire flaws in the wire ropes. The accurate axial and circumferential positions of these broken wire flaws were estimated from the MFL scanning image results. In addition, the proposed TMR-based sensor array was applied to detect the MFL signal induced by slight surface wear defects. A mutual correlation analysis method was used to distinguish the signals caused by the lift-off fluctuation from the MFL scanning image results. The MFL sensor arrays presented in this study provide inspiration for the designing of tailor-made TMR-based circular sensor arrays for cylindrical ferromagnetic structural inspections.
\end{abstract}

\section{Introduction}

In recent years, magnetoresistive (MR) devices have been integrated into minimalistic sensors for nondestructive testing applications $[1,2]$. Compared to inductive coils, the MR-based sensors can operate in ultralow frequency ranges with impressive signal to noise ratios (SNR). With its high spatial resolution and high sensitivity to weak magnetic fields, the MR-based scanning eddy currents or magnetic flux leakage (MFL) sensors can successfully detect the surface or subsurface cracks in conductive or ferromagnetic materials $[3,4]$. As the newest member of the MR family, the tunnel magnetoresistive (TMR) device has been reported to demonstrate superior performance compared to the anisotropic magnetoresistive (AMR) and giant magnetoresistive (GMR) devices [5]. As per the latest publications, TMR-based scanning eddy current systems have the ability to detect surface notches with dimensions as low as a $400 \mu \mathrm{m}$ length, $30 \mu \mathrm{m}$ width, and $30 \mu \mathrm{m}$ depth in titanium alloy samples [6]. The strength of the magnetic field induced by the eddy current generally is located in the limited linear operation range of the
TMR. However, in regard to the magnetic flux leakage (MFL) detection in ferromagnetic materials, the magnetizer needs to be carefully deployed in order to avoid saturation of the TMR in the applied magnetic or flux fields which were induced by the defects. Excitation coils with lower DC currents or surrounding magnetic shield structures are commonly employed to create the proper magnetic environment for TMR devices $[7,8]$.

In our previous research, a single TMR-based MFL detection system was developed for the detection of flaws in hoist wire ropes [9]. The MFL signal induced by a single broken wire $(0.5 \mathrm{~mm}$ in diameter $)$ in a $14 \mathrm{~mm}$ wire rope could be clearly recognized from the oscillation signal which was generated by the twisted rope's surface. In addition, the minimal angular detection range for a single TMR element was estimated, and it was determined that it was insufficient to cover the outside surface of the entire wire rope. Therefore, a circular sensor array needed to be developed for the practical inspection of steel wire ropes.

In this study, TMR devices were used to form circular sensor arrays for detecting the MFL signals induced by 
TABLE 1: Technical parameters of the TMR devices.

\begin{tabular}{lccccc}
\hline Type specification & Sensitive direction & Sensitivity $(\mathrm{mV} / \mathrm{V} / \mathrm{Oe})$ & Dynamic range $(\mathrm{Oe})$ & Linearity range & Hysteresis $(\% \mathrm{FS})$ \\
\hline MMLP57H & In-plane & 4.9 & \pm 90 & \pm 30 & 0.1 \\
MMLH45F & Out-of-plane & 12.0 & \pm 50 & \pm 15 & 1.1 \\
\hline
\end{tabular}

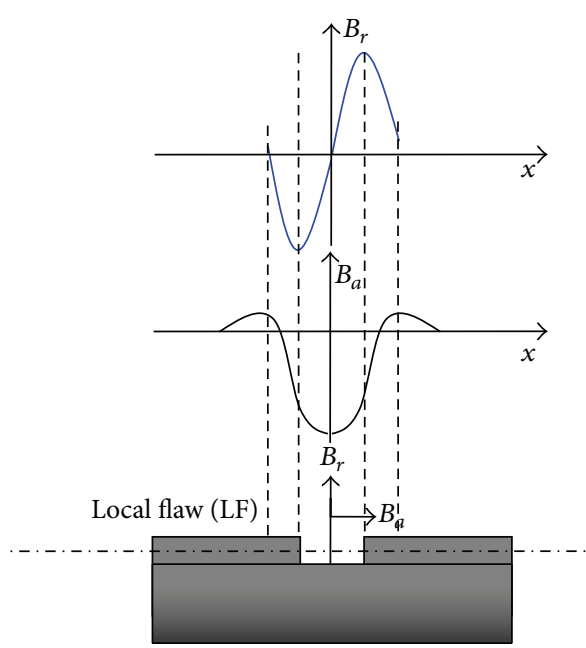

(a)

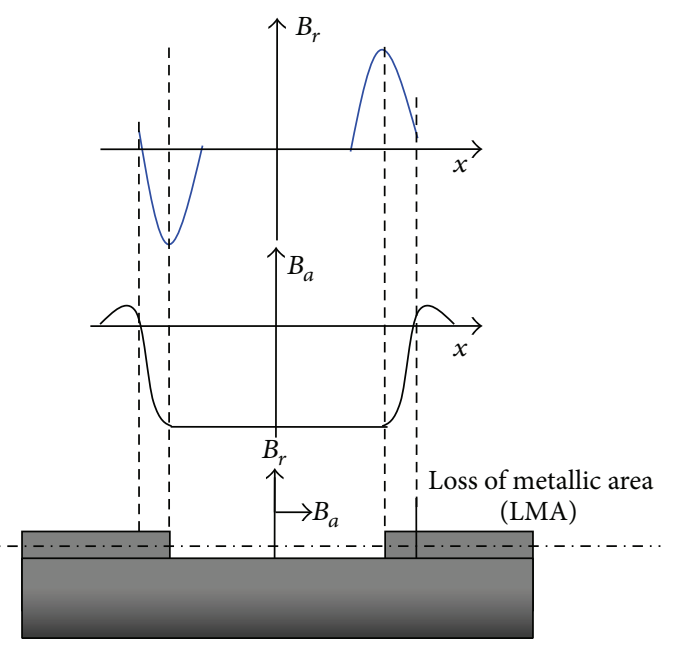

(b)

Figure 1: Axial and radial components of the MFL signal induced by local flaws and loss of metallic area defects.

defects in steel wire ropes with a diameter of $14 \mathrm{~mm}$ and $40 \mathrm{~mm}$, respectively. An electromagnetic magnetizer and a permanent magnetic circuit were deployed for the two sensor arrays. Finally, multiple single-broken wire flaws and wear defect detection, as well as their visualization, were successfully accomplished using the proposed TMR-based sensor array.

\section{TMR-Based Sensor Array for the MFL Method}

2.1. Design of the Circular MFL Sensor Array. Magnetic dipole and finite element methods are commonly used to analyze the induced magnetic flux leakage field (FLL) distribution at a defect in a structure [10]. In this study, the single-broken wire and axial wear defects in wire ropes were the focus. According to the published results on this topic, the components of FLL at both the axial and radial directions of a wire rope are usually studied for defect extent characterizations [11]. Figure 1 shows both the axial and radial component of the FLL induced by local flaws (such as broken wires and pitting defects), along with the loss of metallic area defects (such as wear and corrosion).

In this study, the MFL signal was detected using a magnetic sensor with its sensitivity direction being parallel to the axial or radial direction. The design of a MFL sensor with an optimal compact structure and enhanced sensitivity was a premise to obtain high quality signals. Two methods were commonly employed to improve the MFL signal amplitude. One of the ways was to increase the applied magnetic field, which had the ability to strengthen the FFL. Bulkysized permanent magnet circuits have been designed for this purpose. These types of MFL sensors apply strong dragging effects on wire ropes to cause serious lift-off fluctuation and consequently intense noise is induced to disturb the detected MFL signals. Moreover, the compression effects on the FFL simultaneously enlarge as the applied magnetic field is increased [12]. The creation of a near-zero field zone using magnetic shield has been proven to be an effective way to minimize the compression effects. Another method which is used to improve the MFL amplitude is to employ devices with high sensitivity to the FLL while befittingly reducing the applied magnetic field, so that it may devise a compact sensor which can still output the MFL signal with comparable amplitude. The development of the GMR or TMR based MFL sensors belongs to the latter way to solve this problem. In this study, the TMR devices of MMLP57F and MMLH45F, provided by the MultiDimension Technology Co., Ltd., were used for the circular MFL sensor array. Their technical parameters are listed in Table 1.

The design of an effective magnetizer is the primary task for the construction of a TMR-based MFL sensor. First of all, in the sensitive area of the TMR, the tested sample should be evenly magnetized in the applied magnetic field. Secondly, the strength of the applied magnetic field needs to be carefully selected in order to maximize the TMR's sensitivity to the FLL at the defect. The determination of the accurate strength of the applied magnetic field or FLL inside the magnetic shield is vitally important for the design of an optimal magnetizer. In reality, the strength of the FLL is quite difficult to measure or predict using a finite element method. In this study, an electromagnet which can provide an adjustable magnetic field or a magnetizer with an alterable permanent magnet is prepared for experimental attempts to help determine the 


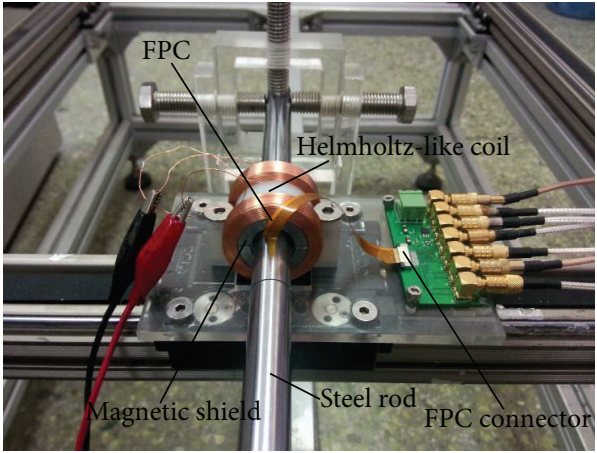

(a)

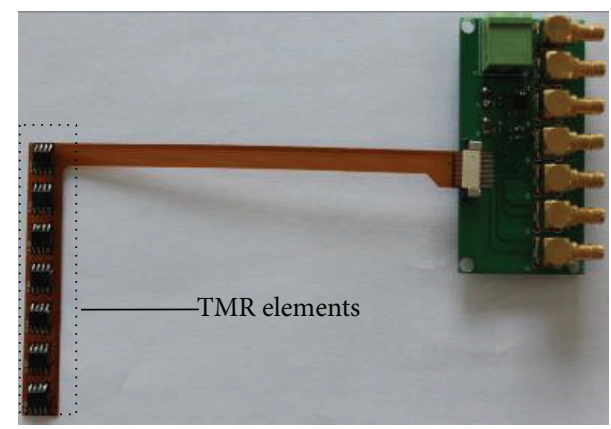

(b)

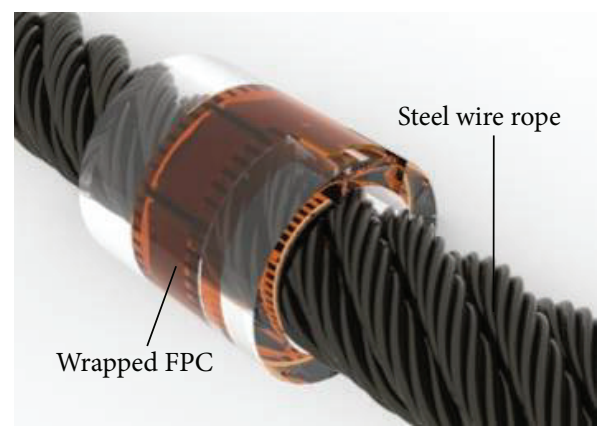

(c)

FIgURE 2: MFL sensor array employing seven TMR elements and a Helmholtz-like coil as a magnetizer.

optimal applied DC current of the electromagnet or the surface induction of the permanent magnet.

In this research study, in order to inspect hoist wire ropes with relatively small diameters, Helmholtz-like coils were used together with magnetic shielding as the magnetizer (Figure 2(a)). An orthogonal test method was employed to aid in the determination of the optimal structural parameters of this magnetizer. As discussed in the authors' recently published study results [9], the available detection angle of a single TMR element (MMLP57H) is approximately 50 degrees, so that a total of seven elements are employed to form a circular sensor array. The TMR elements are then welded onto a flexible printed coil (FPC) strip (Figure 2(b)). After being wrapped around the tested wire rope surface (Figure 2(c)), the entire FPC strip is embedded into the magnetic shield. The entire MFL sensor measures $38 \mathrm{~mm}$ in outer diameter and $26 \mathrm{~mm}$ in height. This can be applied for inspecting wire rope with a diameter less than $14 \mathrm{~mm}$.

The Helmholtz-like coils are wound onto the tested wire ropes. Although the process of coil winding is time consuming, the Helmholtz-like coils are applicable magnetizer for the inspection of wire ropes with small diameters because they have a simplified configuration but provide an evenly distributed and adjustable magnetic field. Thus, in the case of inspecting the wire rope with a diameter of $14 \mathrm{~mm}$, the Helmholtz-like coils are employed as magnetizer for quickly evaluating the feasibility and performance of the TMR-based circular MFL sensor array.

In order to prompt the practical applicability of the MFL sensor for larger lifting wire rope inspection, the abovementioned methodology of designing TMR-based sensor array was transplanted for the purpose of developing a permanent magnet-based magnetizer. This time the MFL sensor array is tailor-made for the lifting wire ropes with a diameter in the range of $38 \sim 42 \mathrm{~mm}$. According to the requirements of the end user, the entire MFL sensor should be in the form of a hollow cylinder, and the height of the cylinder was restricted to less than $50 \mathrm{~mm}$, so that it could be applied to detect the area close to wire rope connectors to shorten the blind inspection range. The developed prototype of the TMRbased MFL sensor array is shown in Figure 3(a). Its height was $46 \mathrm{~mm}$ and its weight was $750 \mathrm{~g}$.

The magnetizer was composed of six branches of yoke circuits and an embedded magnetic shield which had the same shape as that of the first version. The yoke circuits were divided into two identical parts, each of which was mounted and pasted onto a cylindrical plastic holder. Inside each part of the magnetic shields, a flexible printed coil strip equipped with eight TMR elements (MMLH45F) was bent into a C shape to fit the wire rope surface (Figure 3(b)). After the two parts were assembled to form a circular MFL sensor array, the hollow space which held the wire ropes had a diameter of $46 \mathrm{~mm}$. All 16 of the TMR elements were protected by plastic shells with $1 \mathrm{~mm}$ thicknesses. The electrical connections of power supply and the signal output of the TMR elements were printed in the strip (Figure 3(c)). The end of the strip was then connected to a battery which provided the power supply, along with an acquisition card, via an FPC connector.

2.2. Defect Detection Performance Test. The uniformity of the TMR element for sensitivity to an MFL signal determines the performance of the circular sensor array. Any differences 


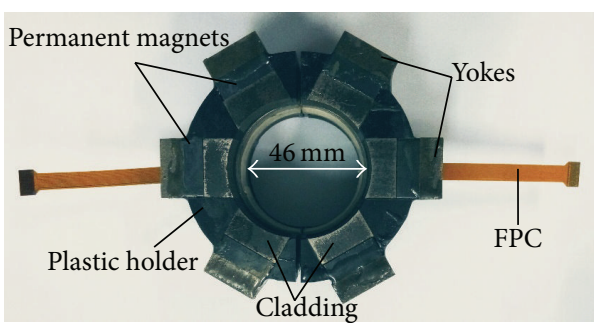

(a)

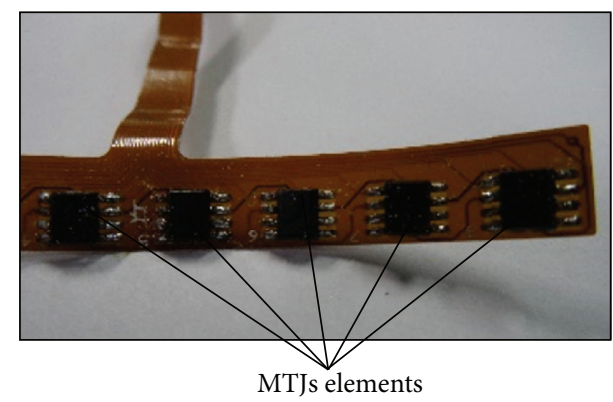

(b)

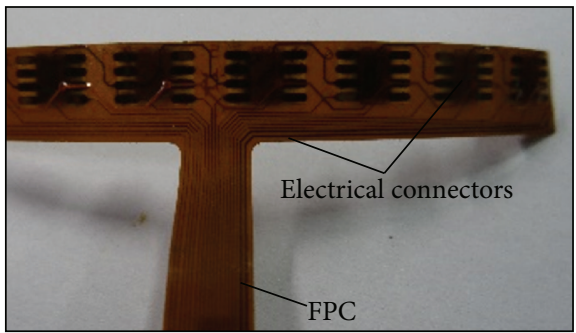

(c)

FIGURE 3: MFL sensor array equipped with 16 TMR elements for practical wire rope inspection applications.

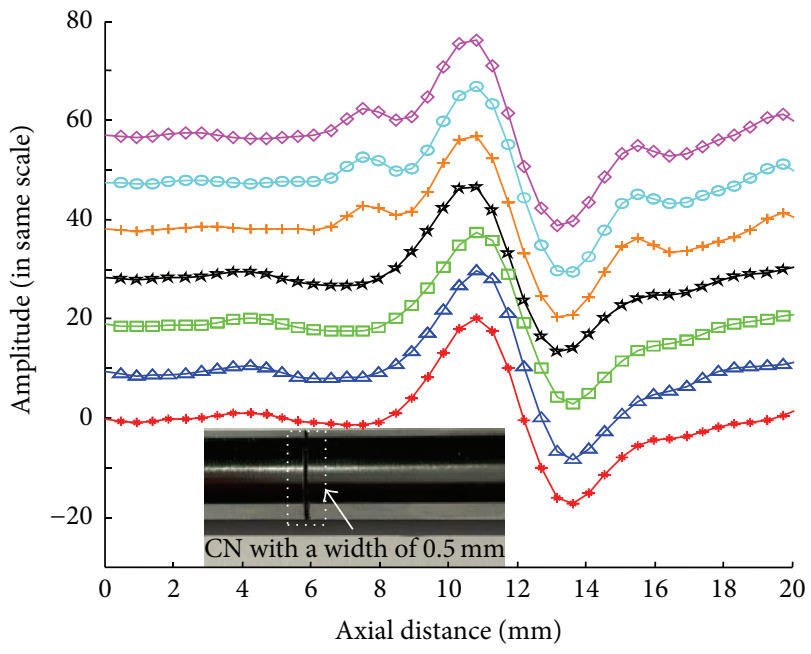

(a)

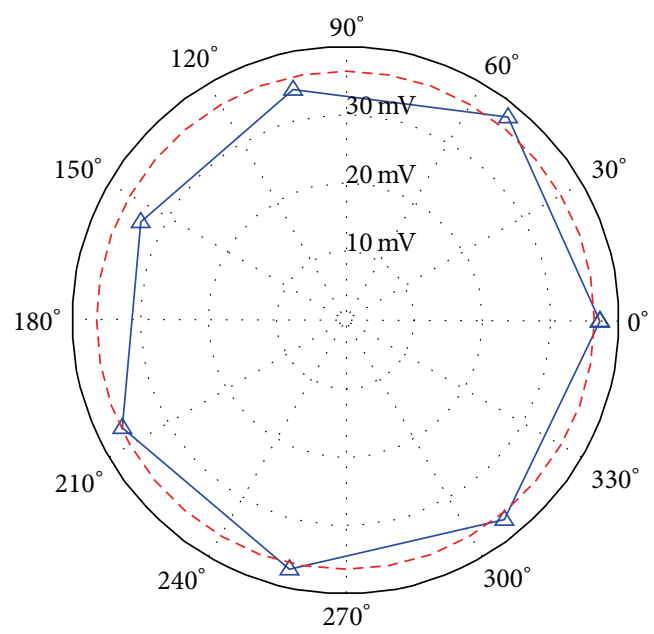

(b)

FIGURE 4: MFL signals detected by the sensor array at a circumferential notch and the estimated peak-to-peak amplitudes of the signals.

may be caused by the variability in TMR's manufacturing and electrical connectors printing procedures. It is well known that an imperfect magnetizer and component assembling errors can magnify the differences among the elements of a circular MFL sensor array.

Figure 2(a) illustrates the experimental setup in this study which was constructed for testing the uniformity of MJT elements in the sensor array. The MFL sensor array scanned along a steel rod with a diameter of $14 \mathrm{~mm}$ at a constant speed. When it passed through a circumferential notch $(\mathrm{CN})$ measuring $0.5 \mathrm{~mm}$ in both width and depth, the output signals of all seven TMR elements were acquired. Third-order bandpass digital Butterworth filter with cutoff frequency of $5 \mathrm{~Hz}$ and $15 \mathrm{~Hz}$ is applied to the received MFL signals. Figure 4(a) shows the band-pass filtered signal waveforms. The sine wave-like anomalies which were centered at approximately $12 \mathrm{~mm}$ in axial distance represented the MFL signal induced by the notch. The peak-to-peak amplitudes of the anomalous waves were estimated and plotted as a polar chart as shown in Figure 4(b).

The coefficient of the variation $\left(C_{v}\right)$ represented the ratio of the standard deviation $(\sigma)$ to the mean $(\mu)$, and it was a useful statistic for evaluating the variation degree of the data series. Smaller value of $C_{v}=\sigma / \mu$ indicates better uniformity among the elements of the data series. The estimated value of $C_{v}$ for the data (Figure 4(b)) was approximately $4.05 \%$, 

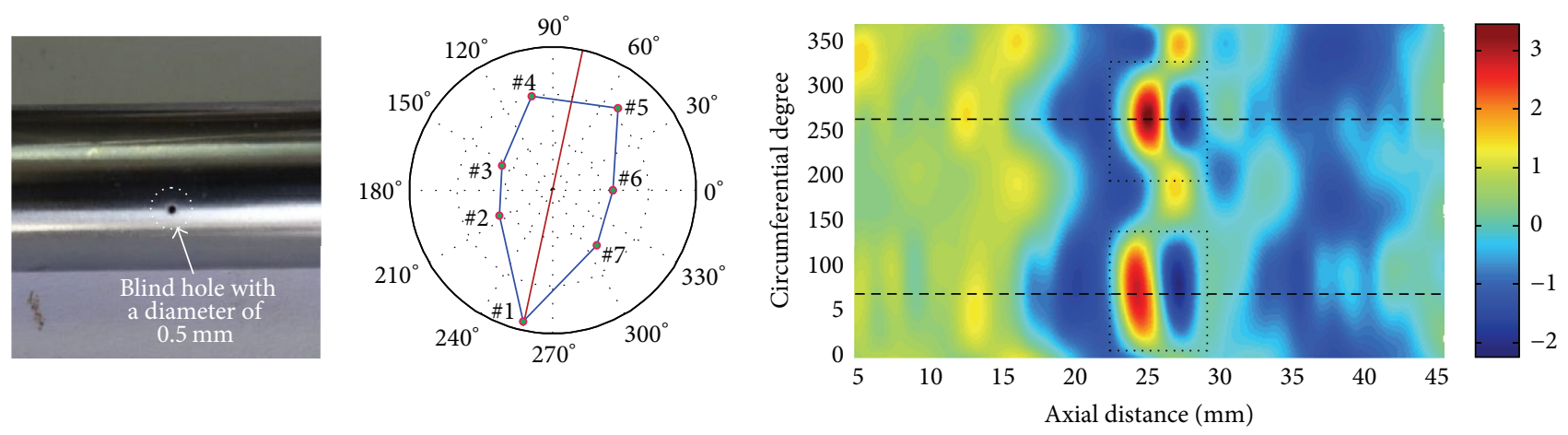

(a)
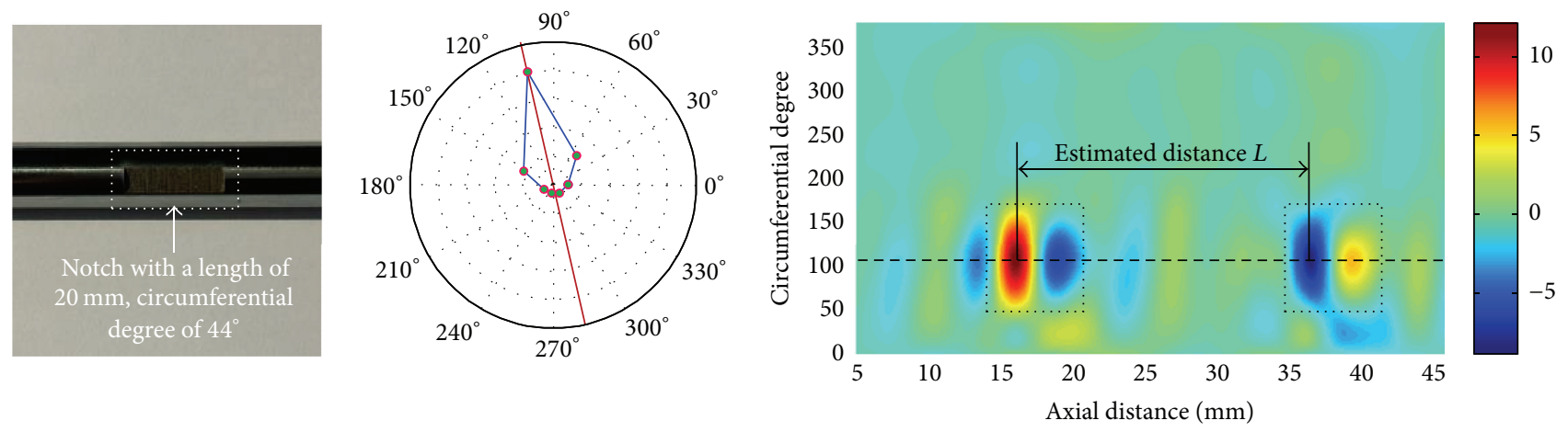

(b)

FIGURE 5: Experimental testing results obtained from a steel rod with two blind holes or an axial notch using the TMR-based sensor array.

which indicates good uniformity in MFL signal detection performance of all the seven TMR elements.

The sensitivity area of a single TMR along a circumferential direction of a wire rope is very limited. When the TMR deviates from the defect location, its output MFL signal amplitude will rapidly decrease. This may lead to a reduction in detection probability of broken wires located at the middle of the two contiguous TMR elements. Figure 5(a) illustrates the results, which can be used to certify these conclusions. A blind hole with a diameter and depth of $0.5 \mathrm{~mm}$ was located in the middle of TMR's elements \#4 and \#5, while another blind hole was located directly beneath the TMR's \#1 element. The amplitudes of the output signals of elements \#4 and \#5 were found to be lower than that of element \#1 by approximately $25 \%$. This may lead to failure in the detection of small flaws that is exactly in the middle of two adjacent TMR elements. Therefore, for the tested wire rope with a given diameter, it is better to arrange TMR elements as much as possible along the circumferential direction of the wire rope to improve the detection probability and circumferential spatial resolution of the sensor array. It should be noted that the plastic holder of the sensor array shown in Figure 2(a) could accommodate up to seven TMR elements.

Compared to the blind holes, slight wear defects with varied loss of metallic area are more difficult to detect using the MFL method. A notch with an axial extension of $20 \mathrm{~mm}$ and a circumferential degree of $44^{\circ}$ was machined in a steel rod with a diameter of $14 \mathrm{~mm}$ for the purpose of simulating the loss of metallic area defects. The maximal depth of the notch was $0.5 \mathrm{~mm}$. Once again, the proposed circular sensor array scanned along the rod, and the received MFL signal amplitudes were plotted as a polar chart as shown in Figure 5(b). A linear interpolation was applied to the signals for the purpose of generating the scanning image for defect visualization. Since the circumferential degree of the notch was concentrated in the sensitive range of a single TMR, in the polar chart the estimated amplitudes distribution is exhibited in obvious directionality. The maximal MFL signal amplitude could be obtained only when one of the TMR elements was placed above the notch. In the scanning image results, the two separated MFL signal indicators could be clearly distinguished. They displayed an opposite feature in a signal phase, which represented a typical axial wear defect. The axial extent of the notch could then be roughly estimated by the distance between these two signal indicators as having a value of $20.16 \mathrm{~mm}$.

\section{Application for Wire Rope Inspections}

The results shown in Figure 5 revealed the excellent performance for defect detection of the proposed TMR-based MFL sensor array (Figure 2) that is developed for inspecting the wire ropes with a diameter less than $14 \mathrm{~mm}$. Although the sensitivity of the TMR elements to circumferential defects is dependent on the orientation of the sensor array, it is expected to be applicable to the sensor array for wire rope inspections, especially in regard to the determination of the defects' axial locations. 


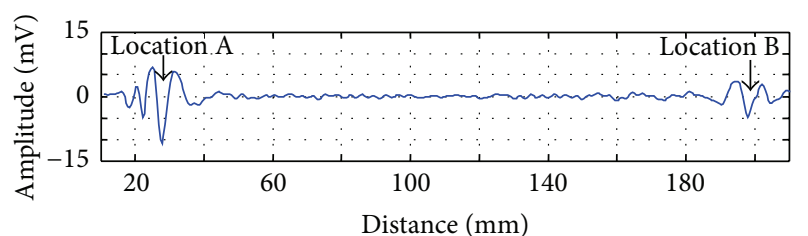

(a)

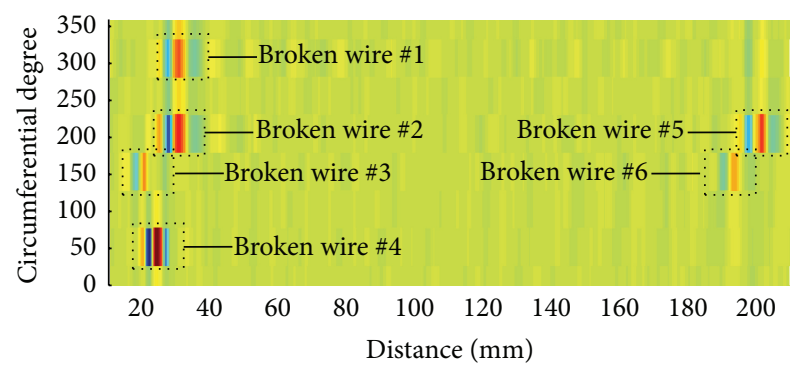

(b)

FIGURE 6: Experimental testing results obtained from wire rope with six broken wires.

Figure 6 shows the results obtained from a defective $6 \times 19+$ IWS wire rope with a diameter of $14 \mathrm{~mm}$. Four and two single-broken wire flaws were cut around locations $A$ and $B$, respectively. At location $A$, broken wires \#1, \#2, $\# 3$, and \#4 were approximately evenly distributed along the circumferential surface. Also, broken wires \#5 and \#6 were close to each other at location $\mathrm{B}$.

In this study, by simply superimposing the MFL signals detected by the seven elements (Figure 6(a)), two anomalous signals were observed approximately near locations A and B. The superimposed MFL signal at locations A and B had SNR of around $15 \mathrm{~dB}$ and $19 \mathrm{~dB}$, respectively. Thus, the existence of the flaws and their axial locations can be quickly identified. More detailed information regarding the defects, including the number of defects and their more accurate locations, could then be estimated in the scanning image results, as shown in Figure 6(b). The indicators near both locations A and $B$ clearly reflected the existence of a total of six artificial broken wire flaws in the wire rope. Furthermore, the broken wire flaws at locations $\mathrm{A}$ and $\mathrm{B}$ were found to be not aligned at the same circumference. This was due to the fact that the broken wire flaws were manually cut at different strands of the wire rope, and the locations were not easy to control at the same circumference.

As illustrated in Figure 7(a), an actual surface wear defect was visible in the wire rope. At least two broken wires were involved in the wear area. When the MFL sensor array scanned along the wear defect, the output signals of the TMR elements were quite complicated, as shown in Figure 7(b). All of the seven elements were able to detect the wear-induced MFL signal, even though the signals varied in shape and peak amplitudes. Unlike in the notch defect case, the scanning image result failed to give the accurate location and extent of the wear defects without any prior knowledge regarding the wear-induced MFL signal features. Herein, the beginning and end positions of the MFL signal were roughly estimated
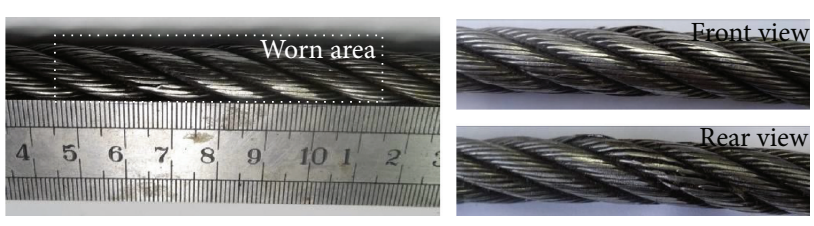

(a)

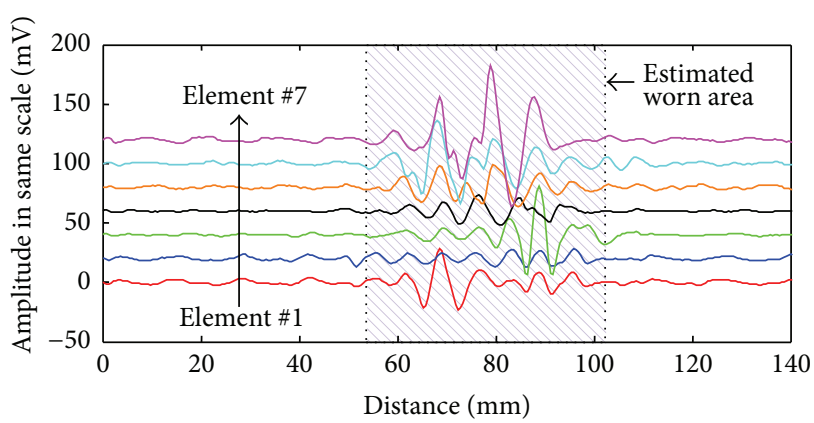

(b)

FIGURE 7: MFL signals induced by an actual surface worn area in the tested wire rope.

as $55 \mathrm{~mm}$ and $102 \mathrm{~mm}$, respectively. The axial extent of the worn area was approximately $47 \mathrm{~mm}$, which was shorter than that of the practical situation in the tested wire rope. The wear defect sizing was a challengeable topic for the MFL method. Since the proposed TMR-based circular MFL sensor array was capable of detecting such slight wear defects, an expected database for restoring the MFL signal induced by the various types of wear defects could be established. Then, the developed advanced signal processing techniques and intelligent signal identification methods could be employed for solving the wear defect sizing problem $[13,14]$.

The lift-off of the sensor to the tested wire rope with a diameter of $14 \mathrm{~mm}$ during the above-mentioned experiments kept constant at $0.5 \mathrm{~mm}$. A controllable scanning apparatus was employed to steadily drag the sensor at constant speed along the tested sample, so that the fluctuation of the lift-off during scanning was minimized.

The second version of TMR-based MFL sensor array was designed for practical wire rope inspection applications. An antiwear shell with a thickness of $1 \mathrm{~mm}$ was placed between the wire rope surface and the TMR protection bobbin. The lift-off of the TMR to the wire rope surface was designed as $3 \mathrm{~mm}$, in order to enable the sensor array to smoothly pass through the wire rope, even if the broken wires jumped to the surface. To simulate the practical situation, the sensor array was manually moved along the wire rope to detect the defects. This time, the lift-off inevitably fluctuated during the scanning process and induced unexpected noised signals.

Figure 8 shows the scanning image results obtained from the $6 \times 19 \mathrm{~s}+\mathrm{FC}$ wire rope with a diameter of $40 \mathrm{~mm}$ used in this study. The superposed result of all sixteen elements' output signals assisted in quickly distinguishing the axial location of defective areas. If the threshold value was selected as $50 \mathrm{mV}$, three suspected defective zones could be observed (Figure 8(a)) which were consistent with the actual situation. 


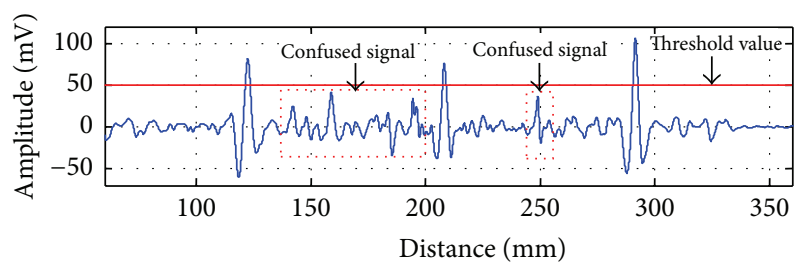

(a)

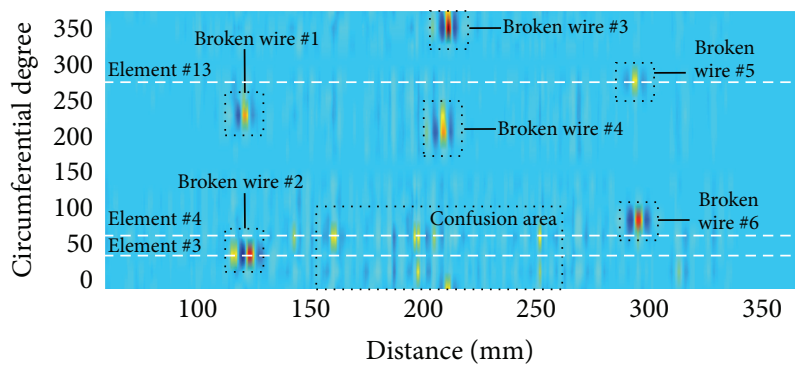

(b)

FIGURE 8: MFL scanning image results obtained from a $40 \mathrm{~mm}$ wire rope with multiple single-broken wire flaws.
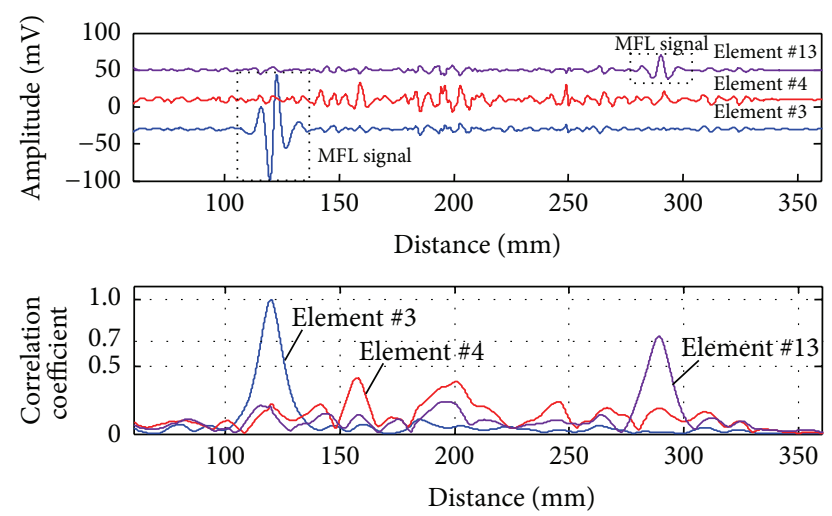

FIGURE 9: Correlation analysis results among the MFL signals received by \#3, \#4, and \#13 elements.

Near each of these three locations, two separated broken wires were cut in a circumferential direction of the wire rope.

Elements \#1 to \#4 received some suspect MFL signals which led to the appearance of a confusion area in the scanning image results. Although these signals had relatively lower amplitudes, it was difficult to distinguish them from the actual MFL signals by simply applying a threshold value to the signal (Figure 8(a)). The typical MFL signal induced by a broken wire can be simulated as the signals having the shapes shown in Figure 1(a). After being filtered, the shapes close to a windowed tone burst signal. Therefore, in the scanning image results, a typical MFL signal was found to have periodical amplitude reversals. This unique feature can be used for MFL signal identification by applying a mutual correlation analysis among the signals.

The signals received by elements \#3, \#4, and \#13 were recalled and plotted, as shown in Figure 9. The signal of element \#3 was used as the standard MFL signal, and the mutual correlation coefficient (MCC) between the signals of elements \#3 and \#4 (or \#13) was estimated. The selfcorrelation coefficient of the signals of element \#3 was also calculated for comparison purposes.

When the MCC was lower than 0.5, it was deduced that the candidate signal could not be treated as a featured standard signal. The maximal MCC between the signals of elements \#3 and \#4 was approximately 0.42 . In this study, there was a tendency to believe that the suspected signal in the confusion area was generated by the fluctuation in sensor lift-off during its passing through the wire rope in the range of $150 \mathrm{~mm}$ to $270 \mathrm{~mm}$. The MFL signal induced by broken wire \#5 had a comparative amplitude as the signal in the confusion area. However, it was highly similar to the typical MFL signal, yielding a value of 0.73 in the MCC. The fluctuation in sensor lift-off is a problem which is quite frequently faced during MFL detection. It was very important to design an effective sensor array carrier structure which enabled the sensor array to move steadily and smoothly along the wire rope being tested.

\section{Conclusions}

Two versions of TMR-based circular MFL sensor arrays and their flaw detection performances were presented in this study. The first version employed Helmholtz-like coils as its magnetizer and seven TMR elements for its feasibility validation. The experimental testing results showed that this TMR-based sensor array could effectively detect slight defects, including blind holes and notches in steel rods. The amplitude deviation of the signals which were separately detected by the seven elements was estimated at $4.05 \%$. The actual axial wear and multiple single-broken wire flaws were also detected by the TMR-based sensor array. Both of the MFL detection results gave an impressive signal to noise ratio (SNR), and the accurate axial and circumferential locations could be estimated from the scanning image results.

For the purpose of developing a TMR-based circular MFL sensor array for practical wire rope inspection, the second version of the MFL sensor array employed a ferrite magnetbased magnetizer and sixteen TMR elements, in order to increase the circumferential resolution to the wire rope. The experimental results obtained from a defective wire rope with a diameter of $14 \mathrm{~mm}$ were presented in this study. The MFL signals induced by six single-broken wire flaws could be clearly identified from the scanning image results. The interference signals caused by the lift-off fluctuations may have formed a confusion area in the scanning image results. However, a simple mutual correlation analysis assisted in distinguishing these from the real MFL signals.

The two versions of the MFL sensor arrays presented in this study provide reference scenarios for the design of tailor-made TMR-based circular sensor arrays for cylindrical ferromagnetic structural inspections. In the future, a sensor array carrier will be designed to form a MFL inspection system, which will be equipped with a compact and highly sensitive TMR-based sensor array.

\section{Competing Interests}

The authors declare that they have no competing interests. 


\section{Acknowledgments}

The work described in this study was fully supported by grants from the National Natural Science Foundation of China (Project nos. 11132002, 11402008, and 51475013); the Beijing Municipal Natural Science Foundation (Project no. 3154030); and the Postdoctoral Science Foundation (no. 2014M560029).

\section{References}

[1] B. Wincheski, J. Simpson, J. Seebo P, and J. Powell, "Highresolution imaging with two-axis orthogonal magneto-resistive sensor based eddy current probe," in Proceedings of the Review of Progress in Quantitative Nondestructive Evaluation, vol. 1430 of AIP Conference Proceedings, pp. 366-372, Burlington, VT, USA, July 2011.

[2] X. Fan, R. Cao, T. Moriyama, W. Wang, H. W. Zhang, and J. Q. Xiao, "Magnetic tunnel junction based microwave detector," Applied Physics Letters, vol. 95, no. 12, Article ID 122501, 2009.

[3] V. Reimund, M. Pelkner, M. Kreutzbruck, and J. Haueisen, "Sensitivity analysis of the non-destructive evaluation of microcracks using GMR sensors," NDT \& E International, vol. 64, pp. 21-29, 2014.

[4] M. Pelkner, M. Blome, V. Reimund, H.-M. Thomas, and M. Kreutzbruck, "Flux leakage measurements for defect characterization using a high precision 3-axial GMR magnetic sensor," AIP Conference Proceedings, vol. 1335, pp. 380-387, 2011.

[5] F. A. Cardoso, L. S. Rosado, F. Franco et al., "Improved magnetic tunnel junctions design for the detection of superficial defects by eddy currents testing," IEEE Transactions on Magnetics, vol. 50, no. 11, Article ID 6201304, 2014.

[6] L. S. Rosado, F. A. Cardoso, F. Franco et al., "Detection of micrometric surface defects in titanium using magnetic tunnel junction sensors," in Proceedings of the 11th European Conference on Non-Destructive Testing (ECNDT '14), Prague, Czech Republic, October 2014.

[7] W. S. Singh, B. P. C. Rao, S. Thirunavukkarasu, and T. Jayakumar, "Flexible GMR sensor array for magnetic flux leakage testing of steel track ropes," Journal of Sensors, vol. 2012, Article ID 129074, 6 pages, 2012.

[8] Y. H. Sun and Y. H. Kang, "A new MFL principle and method based on near-zero background magnetic field," NDT \& $E$ International, vol. 43, no. 4, pp. 348-353, 2010.

[9] B. Wu, Y. J. Wang, X. C. Liu, and C. F. He, "A novel TMR-based MFL sensor for steel wire rope inspection using the orthogonal test method," Smart Materials and Structures, vol. 24, no. 7, Article ID 075007, 2015.

[10] S. M. Dutta, F. H. Ghorbel, and R. K. Stanley, "Dipole modeling of magnetic flux leakage," IEEE Transactions on Magnetics, vol. 45, no. 4, pp. 1959-1965, 2009.

[11] C. Jomdecha and A. Prateepasen, "Design of modified electromagnetic main-flux for steel wire rope inspection," NDT \& $E$ International, vol. 42, no. 1, pp. 77-83, 2009.

[12] Y. H. Sun and Y. H. Kang, "Magnetic compression effect in present MFL testing sensor," Sensors and Actuators A: Physical, vol. 160, no. 1-2, pp. 54-59, 2010.

[13] M. Ravan, R. K. Amineh, S. Koziel, N. K. Nikolova, and J. P. Reilly, "Sizing of 3-D arbitrary defects using magnetic flux leakage measurements," IEEE Transactions on Magnetics, vol. 46, no. 4, pp. 1024-1033, 2010.
[14] D. Mukherjee, S. Saha, and S. Mukhopadhyay, "Inverse mapping of magnetic flux leakage signal for defect characterization," NDT \& E International, vol. 54, pp. 198-208, 2013. 


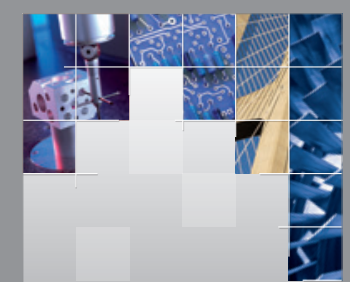

\section{Enfincering}
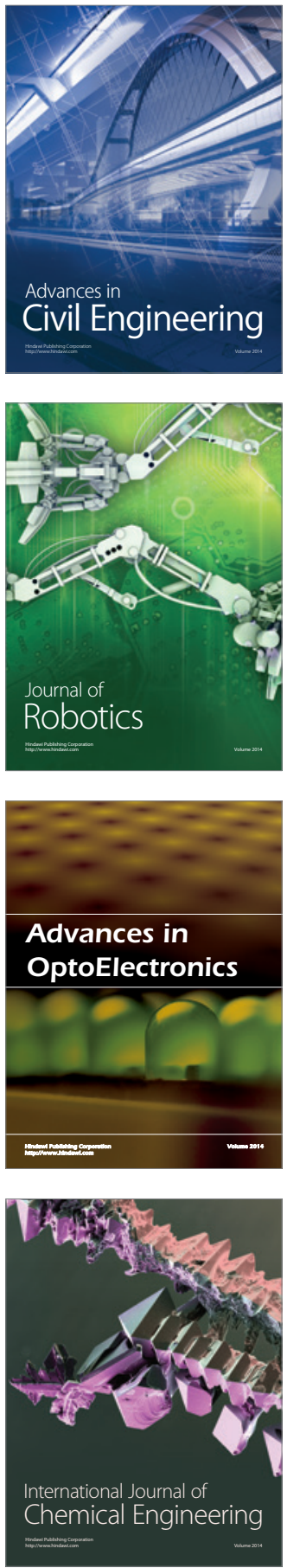

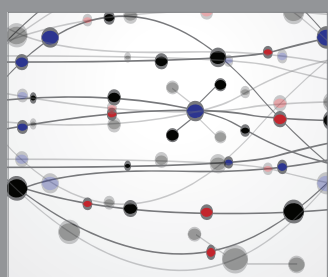

The Scientific World Journal

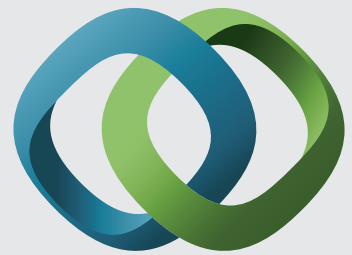

\section{Hindawi}

Submit your manuscripts at

http://www.hindawi.com
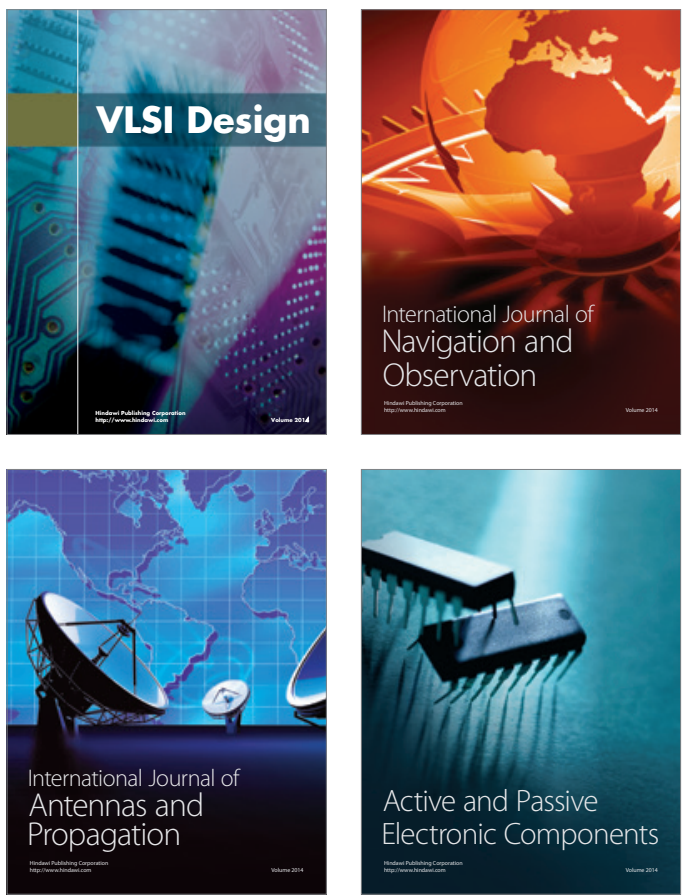
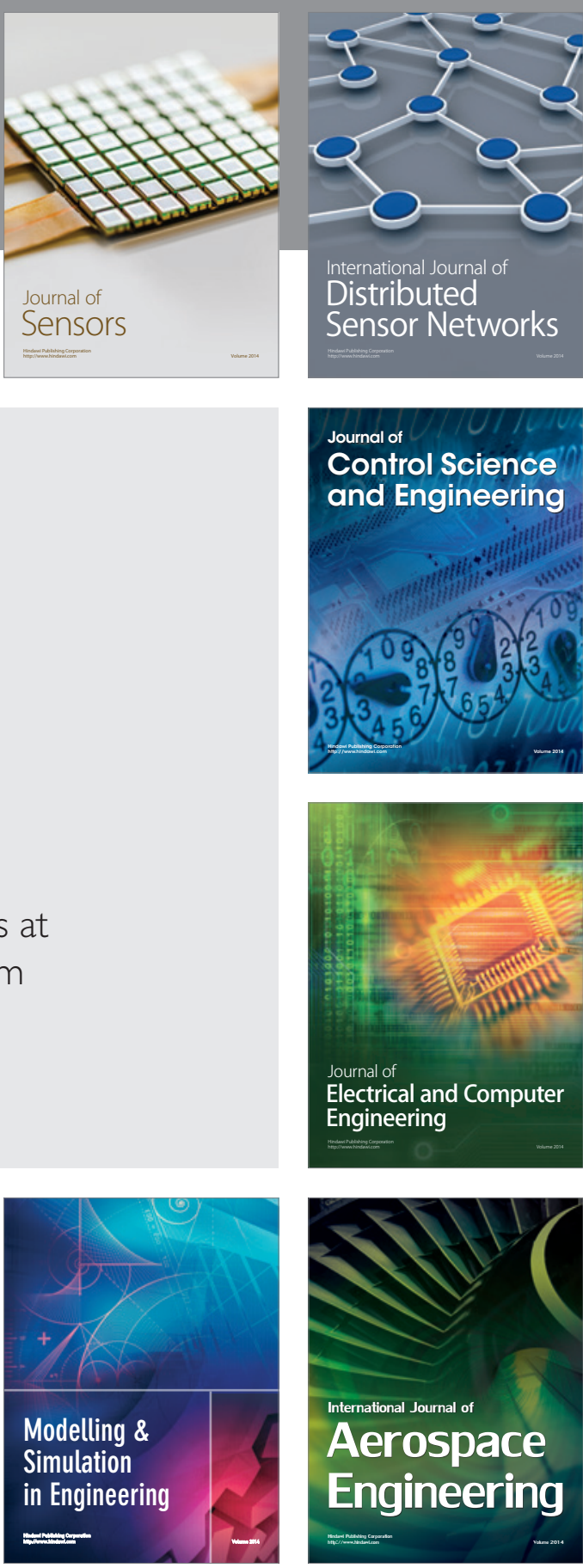

International Journal of

Distributed

Sensor Networks

Journal of

Control Science

and Engineering
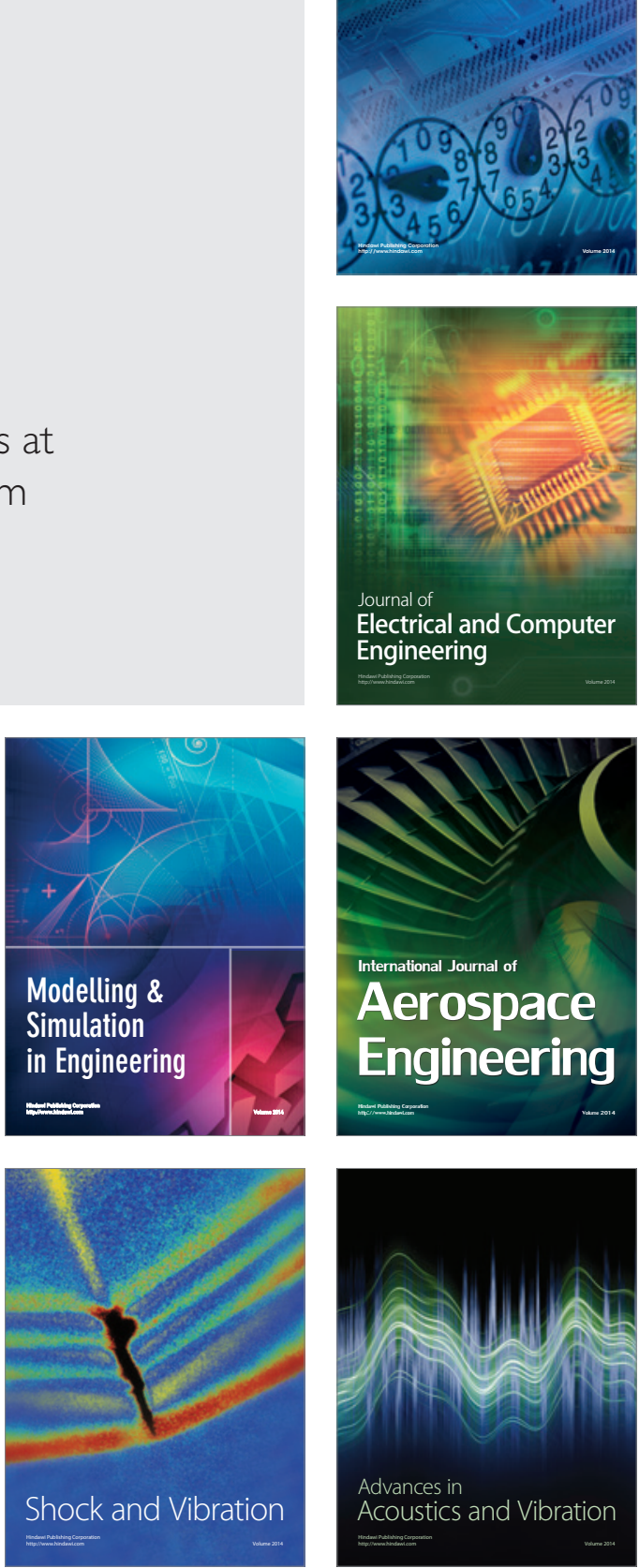